\title{
Acute Effect of Whole-Body Vibration in a Female Dog with Metritis
}

\author{
Ivan Felismino Charas dos Santos, ${ }^{1,2}$, Sheila Canevese Rahal2, Lívia Freire², \\ Carlos Roberto Teixeira ${ }^{2}$, Letícia Rocha Inamassu ${ }^{3}$, Maria Jaqueline Mamprim³, \\ Mayara Viana Freire Gomes ${ }^{2}$ \& Filipe Carrari Isaac Tannus ${ }^{2}$
}

\begin{abstract}
Background: Whole-body vibration is a modality of exercise used in humans for therapeutic purposes or to increase physical performance. In veterinary medicine there are only a few reports on the use of this technology. The Whole-body vibration derivate from vibrating rhythmic movements caused by vibrating platforms. Vibrating platforms are used over 30 years in the treatment and prevention of injuries and other debilitating conditions in humans. This paper aims to describe the first report of a possible spontaneous opening of the cervix in a female dog with purulent metritis by Whole-body vibration using a platform vibration.

Case: A sexually intact female American Pit Bull Terrier dog showed an acute effect after a single session of Wholebody vibration training. Physical examination and physiological parameters were within normal. Slight edema of the vulva was observed without signs of discharge. Complete blood cell count, serum chemistry and urinalysis yielded no significant abnormal findings. An enlarged uterus with content was observed during a transabdominal ultrasound. Despite this, a vibrating-platform session was performed during $15 \mathrm{~min}$. A purulent vulvar discharge was observed 6 min. after Whole-body vibration exercise and remained continuous through session. After the Whole-body vibration exercise, the dog was treated with cephalexin for 15 days. Escherichia coli were isolated from vaginal discharge culture. Seven days after the Whole-body vibration session, no signs of vulvar edema or discharge were observed. A complete blood count, serum chemistry, urinalysis and uterus ultrasound showed no abnormalities. Ovariohysterectomy by minimally invasive technique was performed and was observed cysts in both ovaries. The patient was placed under general anesthesia with isoflurane $/ \mathrm{O}_{2}$. The premedication used was carprofen, morphine sulphate, acepromazine and diazepam. The induction was with propofol. An open ventral midline celiotomy an ovariohysterectomy was performed. Ten days after the surgery the dog showed no abnormality. After recovered from anesthesia, the patient was discharged with meloxican and tramadol for postoperative inflammation and pain management. According to the owner, the immediate postoperative period was without any problems. The histopathology examination confirmed the presence of right ovary cystic and left uterine horn cystic, and uterine hyperplasia. Four months after the surgery, the dog returned for evaluation and the owner reported that patient had been doing well, with normal appetite, normal urination and defecation.

Discussion: The Whole-body vibration is not yet fully understood and it is important that the patient is clinically evaluated before the Whole-body vibration session. Whole-body vibration is a modality of exercise used in humans for therapeutic purposes or to increase physical performance. Otherwise, in veterinary medicine there are only a few reports on the use of this technology. In the present report there were no clinical signs that indicated uterine infection. In human patients there are reports that showed any clinical signs of diseases before use the Whole-body vibration. The ultrasound exam had been done because was part of another study and showed an enlarged uterus. Therefore, the cervix opening after a single Wholebody vibration training may be considered a positive side effect of Whole-body vibration in female dogs since this event helped to diagnose the disease. Although, the effects of WBV on reproductive organs and endocrine system are not clear.
\end{abstract}

Keywords: canine, vibration, uterus, ultrasound, vibration platform.

${ }^{1}$ Pós-doutorando (Bolsista Fapesp). ${ }^{2}$ Departamento de Cirurgia e Anestesiologia Veterinária (DCAV) \& ${ }^{3}$ Departamento de Reprodução Animal e Radiologia Veterinária (DRARV), Faculdade de Medicina Veterinária e Zootecnia (FMVZ), Universidade Estadual Paulista (UNESP), Botucatu, SP, Brazil. CORRESPONDENCE: I.F.C. Santos [ivansantos7@ hotmail.com - Tel.: +55 (14) 3880-2022]. Faculdade de Medicina Veterinária e Zootecnia - UNESP. Distrito de Rubião Junior, s/n. CEP 18618-681 Botucatu, SP, Brazil. 


\section{INTRODUCTION}

Whole-body vibration (WBV) is a modality of exercise used in humans for therapeutic purposes or to increase physical performance $[1,8]$. In veterinary medicine there are only a few reports on the use of this technology $[2,5,12]$. Even in human medicine the beneficial effects and safety of vibration training remain controversial [1,11], as well as if all tissues and organs would be applicable to receive the vibration [11]. The aim of the current report was to describe an acute effect after a single session of WBV training in a dog that presented with purulent metritis.

\section{CASE}

The study was approved by the Ethics Committee of the School of Veterinary Medicine and Animal Science - University Estadual Paulista (UNESP) ( $n^{\circ}$. 042/2014-CEUA). The owner gave written consent for his dog participation in this report case. A 2-year-old, $32 \mathrm{~kg}$, sexually intact female American Pit Bull Terrier dog that was a part of a Whole-body vibration (WBV) study at our Veterinary School, showed an acute effect after a single session of training. On physical examination, the dog had a good body condition, and the physiological parameters (rectal temperature, heart rate and respiratory rate) were within normal limits. Slight edema of the vulva was observed, but without signs of discharge. The owner reported that dog had spontaneous and non-complicated parturition 12 weeks previously, and showed one episode of mucoid vaginal discharge 2 weeks ago. A complete blood cell count (CBC), serum chemistry (alanine aminotransferase ALT, aspartate aminotransferase - AST, blood urea nitrogen - BUN, creatinine - CREAT, alkaline phosphatase, total protein, albumin, globulin and creatine kinase - CK), and urinalysis yielded no significant abnormal findings. A transabdominal ultrasonography was performed using an Esaote ultrasound machine ${ }^{1}$ with multifrequencial convex transducer $(1-8 \mathrm{MHz})$ and an enlarged uterus $(0.79 \mathrm{~cm})$ with hypoechoic luminal content was identified (Figure 1).

Despite this, a vibrating-platform session using a vibrating platform ${ }^{2}$ with dimensions of $92 \mathrm{~cm}$ (length) X $62 \mathrm{~cm}$ (width) and $16 \mathrm{~cm}$ (height) was performed at the frequency of $30 \mathrm{~Hz}$ for $5 \mathrm{~min}$, followed by $50 \mathrm{~Hz}$ for $5 \mathrm{~min}$, and finishing with $30 \mathrm{~Hz}$ for $5 \mathrm{~min}$, with dog standing up on all four feet (Figure 2). The acceleration varied from 12 to $40 \mathrm{~m} / \mathrm{s}^{2}$, amplitude from 1.7 to
$2.5 \mathrm{~mm}$ and peak displacement varied from $3.4 \mathrm{~mm}$ to $5 \mathrm{~mm}$. A purulent vulvar discharge was observed $6 \mathrm{~min}$. following the exposure to WBV (Figure 3) and remained continuous throughout the session. Immediately after the WBV session, a transabdominal ultrasonography was performed and showed a slight decrease of the uterus $(0.73 \mathrm{~cm})$ and intrauterine hypoechoic luminal content (Figure 4).

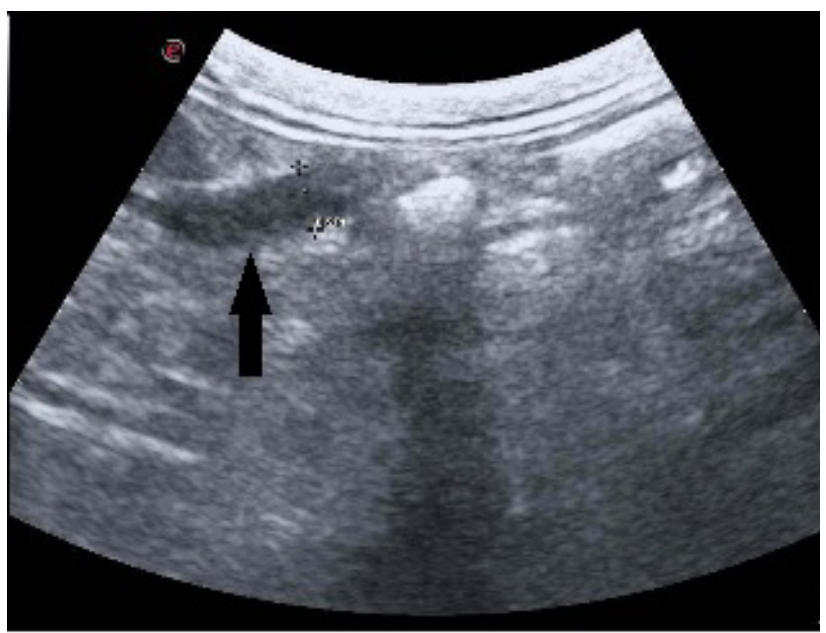

Figure 1. Sagittal ultrasound image (convex 1-8 MHz scan head) showing an enlarged left horn with hypoechoic luminal content (arrow) suggestive of purulent metritis.

The dog was treated with cephalexin, 25 $\mathrm{mg} / \mathrm{kg}$ body weight, orally, every $12 \mathrm{~h}$, for 15 days. Escherichia coli were isolated from vaginal discharge culture. Seven days after the WBV session, no signs of vulvar edema or discharge were observed. A CBC, serum chemistry (ALT, AST, BUN, CREAT, alkaline phosphatase, total protein, albumin, globulin and CK) and urinalysis showed no abnormalities. Ultrasound exam showed no evidence of intrauterine luminal content. However, ovariohysterectomy was elected by the owner in order to reduce risk for the development of pyometra.

The patient was placed under general anesthesia. The premedication was: carprofen $4.4 \mathrm{mg} \mathrm{kg}$ subcutaneously (SC), morphine sulphate $0.5 \mathrm{mg} / \mathrm{kg}$ $\mathrm{SC}$, acepromazine $0.05 \mathrm{mg} / \mathrm{kg} \mathrm{SC}$, diazepam $0.3 \mathrm{mg} /$ $\mathrm{kg}$ intravenously (IV). The induction was with propofol $6 \mathrm{mg} / \mathrm{kg}$ (IV), and maintenance with isoflurane $/ \mathrm{O}_{2}$. The abdominal region was clipped and scrubbed with an alcoholic chlorhexidine solution. An open ventral midline celiotomy an ovariohysterectomy by minimally invasive technique was performed. Both uterine 
horns were moderately thickened, whereas cysts were found in both ovaries and left uterine horn (Figure 5).

The muscular layer was closed with Sultan sutures using 2-0 nylon and subcutaneous layer was closed with simple continuous suture with 2-0 polydioxanone. The skin edges were opposed with interrupted simple sutures using 3-0 nylon. The ovaries and uterus was placed in formalin and submitted for histopathology exam. After recovered from anesthesia, the patient was discharged with meloxican, $0.1 \mathrm{mg} / \mathrm{kg}$, orally, every $24 \mathrm{~h}$, for 5 days, and tramadol, $1 \mathrm{mg} / \mathrm{kg}$, orally, every $8 \mathrm{~h}$, for 5 days, for postoperative inflammation and pain management. The surgical site was treated with healing ointment. According to the owner, the immediate postoperative period was without any

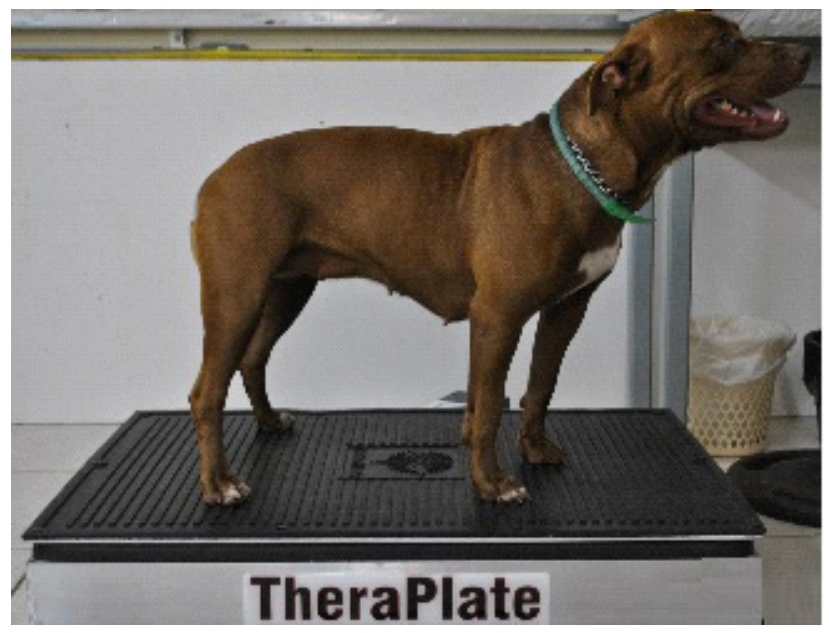

Figure 2. Dog standing up on all four feet positioned in the center of the vibrating platform with dimensions of $92 \mathrm{~cm}$ (length) X $62 \mathrm{~cm}$ (width) and $16 \mathrm{~cm}$ (height) with limbs parallel and located at predetermined fixed point.

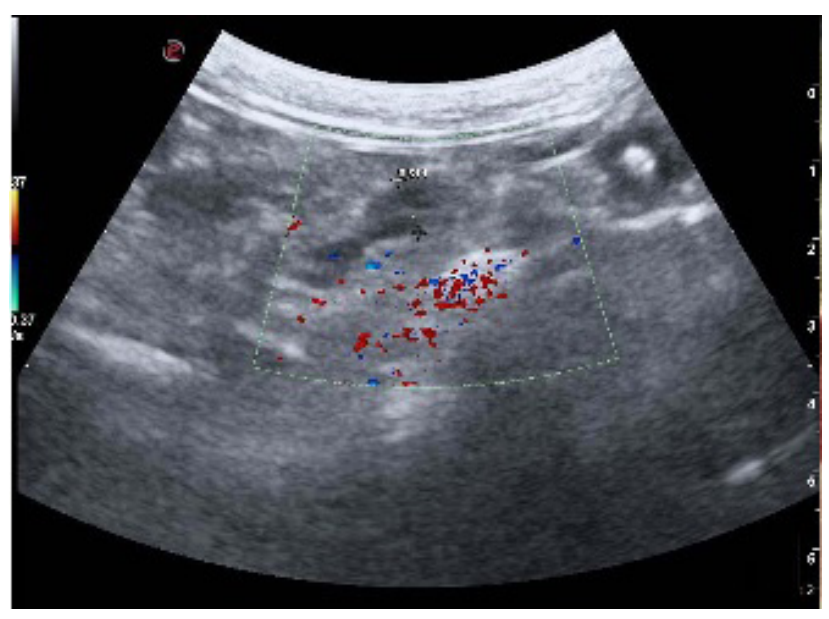

Figure 4. Sagittal ultrasound image (convex 1-8 MHz scan head) showing a slight decrease of the uterus and intrauterine hypoechoic luminal content. problems. Ten days after the surgery was performed a complete blood count, serum chemistry (ALT, AST, BUN, CREAT, alkaline phosphatase, total protein, albumin, globulin and CK) and urinalysis presented results within normal limits. The surgical site healed appropriately.

The histopathology examination confirmed the presence of right ovary cystic and left uterine horn cystic, and uterine hyperplasia. Four months after the surgery, the dog returned for evaluation. The owner reported that patient had been doing well, with normal appetite, normal urination and defecation. The complete blood count, serum chemistry (ALT, BUN, CREAT, total protein, albumin, globulin) and urinalysis and all tests were within normal limits.

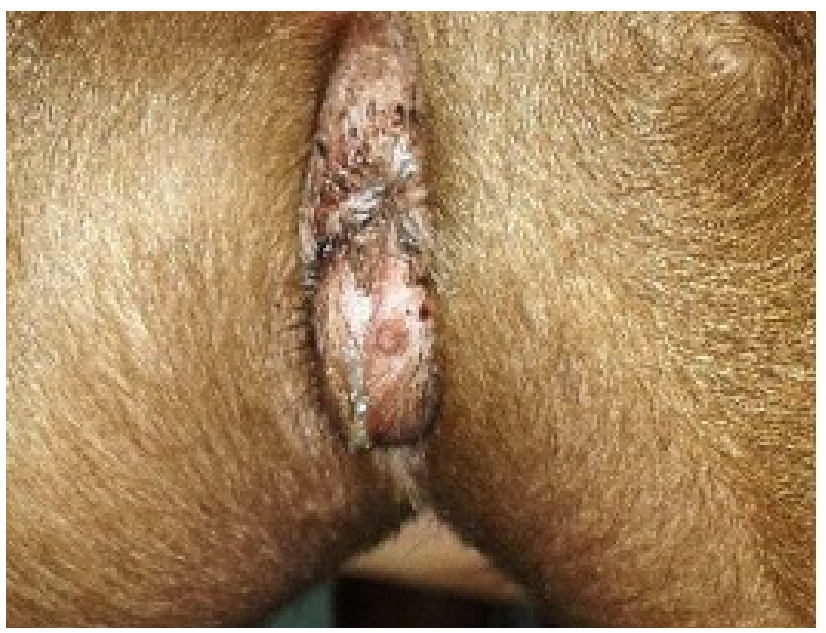

Figure 3. Image showing a purulent vulvar discharge 6 min following the WBV exercise with "TheraPlate" vibratory platform.

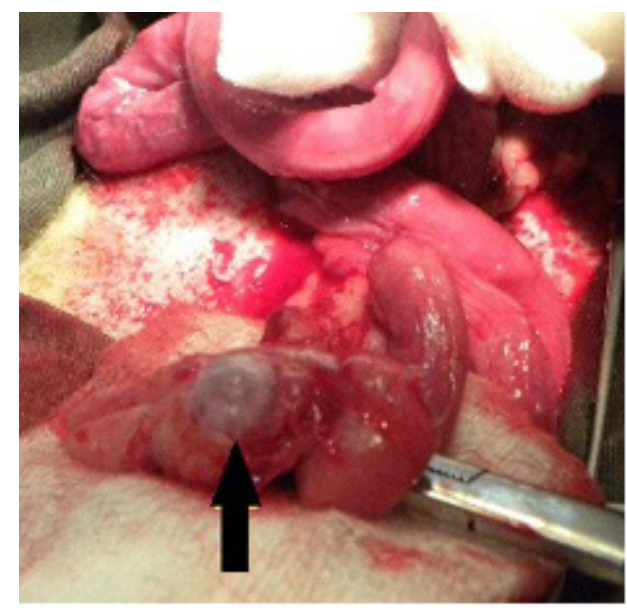

Figure 4. Image showing cyst in right ovary (arrow) and uterine horns moderately thickened detected during ovariohysterectomy. 


\section{DISCUSSION}

Whole-body vibration (WBV) is a modality of exercise used in humans for therapeutic purposes or to increase physical performance $[1,8]$. In veterinary medicine there are only a few reports on the use of this technology in horses [2], in dogs [5] and in penguins [12]. Even in human medicine the beneficial effects and safety of vibration training remain controversial as well as if all tissues and organs would be applicable to receive the vibration $[1,11]$.

Since the biological and mechanical effects of WBV are not yet fully understood, the patient's clinical history must be evaluated before the exam. In the present report, except for one discharge episode, there were no clinical signs that indicated uterine infection. The ultrasound exam had been done because was part of another study and showed an enlarged uterus. In human patients there are reports that showed a healthy elite athlete (steeplechase runner) suffered episodes of hematuria after WBV training (five repetitions of $1 \mathrm{~min}$ at $30 \mathrm{~Hz}$ ) that was supposed to have occurred due to bladder injury produced by repeated impact [4]. On the other hand, a female amateur athlete with asymptomatic nephrolithiasis in association with hydronephrosis presented significant morbidity $12 \mathrm{~h}$ after a single 5 min session of WBV training [9]. A spontaneous vitreous hemorrhage has been described in human two weeks after starting WBV training for a period of 20 min twice a week as well as after a session of WBV training [6].

In the current report case, the cervix opening that occurred after a single WBV training may be considered a positive side effect, since this event helped to diagnose the disease. Although, the effects of WBV on reproductive organs and endocrine system are not clear [3]. A study on pregnant rats exposed to
WBV under a high vibration acceleration rate found changes in uterine and ovarian function along with a decrease in uterine blood flow that was attributed to a reduced prostaglandin E2 concentration. This decrease indicates that vibration can cause ovarian and luteal dysfunction [10].

A wide variety of WBV protocols can be established considering the combinations of frequencies and amplitudes, along with exposure time and direction $[1,7,8]$. In addition, the WBV devices have differences such as type of vibratory stimulation, frequency range, and amplitude [8]. All these parameters affect the biological response to vibration [7].

Vibrating platform protocols for dogs have not yet been described nevertheless a WBV study in kidneys dopplerfluxometry in dogs using the same protocol of the present report showed no alterations [5]. Thus, in the present case report the WBV protocol was based on descriptions provided by the manufacturer for human patients [13], and apparently was sufficient to opening the cervix in a medium-sized dog. However, further investigation is necessary to elucidate the mechanism. To the best of our knowledge, this is the first report of a possible spontaneous opening of the cervix in a female dog with purulent metritis by means of WBV.

\section{MANUFACTURERS}

${ }^{1}$ Esaote Healthcare. São Paulo, SP, Brazil.

${ }^{2}$ TheraPlate Revolution. Weatherford, TX, USA.

Acknowledgments. State of São Paulo Research Foundation (FAPESP) for Post-Doctoral Fellowship (2014/09683-6) of Ivan F.C. Santos, and Brazilian National Council for Scientific and Technological Development (CNPq) [00710/2013-5].

Declaration of interest. The authors report no conflicts of interest. The authors alone are responsible for the content and writing of the paper.

\section{REFERENCES}

1 Cardinale M. \& Wakeling J. 2005. Whole body vibration exercise: are vibrations good for you?. British Journal of Sports Medicine. 39: 585-589.

2 Carstanjen B., Balali M., Gajewski Z., Furmanczyk K., Bondzio A., Remy B. \& Hartmann H. 2013. Short-term whole body vibration exercise in adult healthy horse. Polish Journal of Veterinary Sciences. 16: 403-405.

3 Dovio A., Roveda E., Sciolla C., Montaruli A., Raffaelli A., Saba A., Calogiuri G., De Francia S., Borrione P., Salvadori P., Carandente F. \& Angeli A. 2010. Intense physical exercise increases systemic 11 beta-hydroxysteroid dehydrogenase type 1 activity in healthy adult subjects. European Journal of Applied Physiology. 108: 681-687.

4 Franchignoni F., Vercelli S. \& Ozçakar L. 2013. Hematuria in a runner after treatment with whole body vibration: a case report. Scandinavian Journal of Medicine \& Science in Sports. 23: 383-385. 
5 Freire L., Rahal S.C., Santos I.F.C., Teixeira C.R., Inamassu L.R. \& Mamprim M.J. 2015. Renal resistive index of adult healthy dogs submitted to short-term whole-body vibration exercise. Asian Journal of Animal and Veterinary Advances. 10(11): 797-802.

6 Gillan S.N., Sutherland S. \& Cormack T.G. 2011. Vitreous hemorrhage after whole-body vibration training. Retinal Cases and Brief Reports. 25: 130-131.

7 Jordan M.J., Norris S.R., Smith D.J. \& Herzog W. 2005. Vibration training: an overview of the area, training consequences, and future considerations. The Journal of Strength and Conditioning Research. 19: 459-466.

8 Madou K.H. \& Cronin J.B. 2008. The effects of whole body vibration on physical and physiological capability in special populations. Hong Kong Physiotherapy Journal. 26: 24-38.

9 Monteleone G., De Lorenzo A., Sgroi M., De Angelis S. \& Di Renzo L. 2007. Contraindications for whole body vibration training: a case of nephrolithiasis. The Journal of Sports Medicine and Physical Fitness. 47: 443-445.

10 Nakamura H., Ohsu W., Nagase H., Okazawa T., Yoshida M. \& Okada A. 1996. Uterine circulatory dysfunction induced by whole-body vibration and its endocrine pathogenesis in the pregnant rat. European Journal of Applied Physiology and Occupational Physiology. 72: 292-296.

11 Prisby R.D., Lafage-Proust M.H., Malaval L., Belli A. \& Vico L. 2008. Effects of whole body vibration on the skeleton and other organ systems in man and animal models: What we know and what we need to know. Ageing Research Reviews. 7: 319-329.

12 Santos I.F.C., Sakata S., Rahal S.C., Nascimento C.L., Melchert A. \& Texeira C.R. 2016. Plantar Thermographic evaluation a short-term whole-body vibration magellanic penguins with and without bumblefoot. Asian Journal of Animal and Veterinary Advances. 11(5): 309-313.

13 Theraplate Revolution. 2012. Theraplate Revolution: A revolution for horses, people and companion animals. [Fonte: http://www.theraplate.com/]. [Last accessed November 03, 2014]. 